\title{
Tuberculosis of the parotid lymph nodes: clinical and imaging features
}

This article was published in the following Dove Press journal: Infection and Drug Resistance

\author{
Dan Zhang ${ }^{1,2}$ \\ Xiaojiao $\mathrm{Li}^{2,3}$ \\ Hua Xiong ${ }^{2,3}$ \\ Chao Yang ${ }^{2,3}$ \\ Fajin Lv' \\ Xianlong Huang ${ }^{2,3}$ \\ Qi Li' \\ Zhuoyue Tang ${ }^{2,3}$ \\ Tianyou Luo'
}

'Department of Radiology, The First Affiliated Hospital of Chongqing Medical University, Chongqing 400016, China; ${ }^{2}$ Department of Radiology, Chongqing General Hospital, University of Chinese Academy of Sciences, Chongqing 4000 I4, China; ${ }^{3}$ Molecular and Functional Imaging Laboratory, Chongqing General Hospital, University of Chinese Academy of Sciences, Chongqing 4000 I4, China
Correspondence: Zhuoyue Tang Department of Radiology, Chongqing General Hospital, University of Chinese Academy of Sciences, 104 Pipashan Main St, Yuzhong District, Chongqing 4000I4, China

Email zhuoyue_tang@cqmu.edu.cn

Tianyou Luo

Department of Radiology, The First Affiliated Hospital of Chongqing Medical University, I Youyi Road, Yuanjiagang, Yuzhong District, Chongqing 4000I6,

China

Email Itycqfs@126.com
Objectives: To characterize clinical, computed tomography (CT) and magnetic resonance imaging (MRI) features of tuberculosis (TB) of the parotid nodes.

Materials and methods: $C T(n=21)$ and $M R(n=7)$ images, and clinical data from 25 patients with $\mathrm{TB}$ of the parotid nodes were retrospectively analyzed by two experienced radiologists who reached consensus.

Results: Younger patients (aged $<50$ years) accounted for $72 \%$. Eighty percent of patients were asymptomatic, and had no history of TB exposure. According to clinical and imaging findings, $64 \%$ and $60 \%$ patients were misdiagnosed as having tumors, respectively. A total of 43 lesions were identified. Thirty-eight (88.4\%) lesions involved the superficial lobe. Fourteen (56\%) cases had multiple lesions. There were four types of changes in the parotid fascia: local thickening $(40 \%, n=10)$; local rupture with thickened adjacent skin $(28 \%, n=7)$; focal bulge $(20 \%, n=5)$; and no changes $(12 \%, \mathrm{n}=3)$. Cervical lymphadenopathy was seen in 14 out of 25 cases $(56 \%)$. The lesions were contrast-enhanced in four patterns on CT images: homogeneous enhancement $(37.1 \%, \mathrm{n}=13)$, irregular cyst-like enhancement $(37.1 \%, \mathrm{n}=13)$, thick-walled ring enhancement $(14.2 \%, n=5)$, and garland-like enhancement $(11.4 \%, n=4)$. On MRI, the signal intensity of lesions was isointense on T1-weighted image, hyperintense on T2-weighted image, markedly hyperintense on diffusion-weighted imaging, and low on the apparent diffusion coefficient map. The surrounding parotid parenchymal edema was identified clearly on coronal MR images.

Conclusion: TB of the parotid nodes tend to simulate tumors clinically and radiologically. Their preferential sites are the superficial lobe. In young patients with positive purified protein derivative skin test and lesions accompanied by cervical lymphadenopathy, changes in the parotid fascia and parotid parenchymal edema adjacent to the lesions on CT and MRI may be helpful in the diagnosis and to facilitate differential diagnosis.

Keywords: tuberculosis, parotid nodes, computed tomography, magnetic resonance imaging

\section{Introduction}

Tuberculosis (TB) is an infectious disease caused by Mycobacterium tuberculosis, which can affect any organ. Lymph node tuberculosis (LNTB) is considered the most common type of extrapulmonary TB in developing countries, with an estimated incidence of approximately $28 \% .^{1-5}$ Tuberculous involvement of the parotid lymph node is extremely rare, even in countries where TB is widespread. ${ }^{6-8}$ The diagnosis of LNTB is not always easy because of its rarity and similarities in the presentation with parotid tumors. ${ }^{9}$

It is generally misdiagnosed as a tumor by clinicians and radiologists, with most cases undergoing unnecessary surgeries. ${ }^{10,11}$ Imaging modalities for evaluating parotid 
lesions include ultrasound (US), computed tomography (CT), and magnetic resonance imaging (MRI). Correct clinical analysis and radiological interpretation is helpful in differentiating tuberculous involvement from neoplasms. ${ }^{12}$

The clinical and pathological features of approximately 100 cases of parotid TB have been reported in the English literature. ${ }^{13}$ Only two case reports mentioning the MR manifestations have been presented previously., ${ }^{9,14}$ Owing to its rarity, its clinical and imaging features have not been fully described yet. The purpose of our study was to critically assess our experience in 25 patients to detect specific clinical and imaging findings and with great effort improve diagnostic accuracy and avoid surgical exploration.

\section{Patients and methods}

\section{Patients}

The local institutional ethical review boards approved this retrospective study and the patients signed informed consent. Between January 2008 and November 2016, a total of 25 patients (eleven men, 14 women; age range: 13-76 years; average age: 39.7 years) with pathologically proven TB of parotid nodes were enrolled in this study. CT $(n=21)$ and MR ( $n=7)$ images of these 25 patients were retrospectively reviewed. These patients met the following inclusion criteria: 1) all patients complained of lumps in the pretragal area or below the ear; 2) the patients underwent dynamic contrastenhanced CT and/or MR scan of the neck which showed lumps located in the parotid gland; 3 ) the patients were pathologically diagnosed by surgical resection or biopsy; 4) the CT and MR images were of adequate quality for the diagnosis.

\section{Imaging techniques}

Among the 25 TB patients, CT scanning was performed in 21 patients and MRI in seven patients. Three patients underwent both $\mathrm{CT}$ and MR examinations. Five patients underwent post-contrast T1-weighted imaging.

CT scans were performed using a 64-multidetector scanner (LightSpeed VCT; GE Healthcare, Waukesha, WI, USA) with the following parameters: $120 \mathrm{kVp} ; 150 \mathrm{~mA}$; time, 0.6 seconds; pitch, 0.984; slice thickness and interval for axial images, $5 \mathrm{~mm} / 5 \mathrm{~mm}$. The scanning ranged from skull to rib cage level. Twenty-one patients underwent contrast-enhanced CT scanning with a total of $80-100 \mathrm{~mL}$ of $(1.35 \mathrm{~mL}$ per $\mathrm{kg}$ of bodyweight) nonionic iodinated contrast material $(320 \mathrm{mg} /$ mL; Iopamidol, Shanghai Bracco Sine Pharmaceutical Co., Ltd., Shanghai, China) at an injection rate of $3.0 \mathrm{~mL} / \mathrm{s}$, followed by $50 \mathrm{~mL}$ of saline solution via a power injector. The contrast-enhanced CT images were obtained during arterial and balanced phases at 30,60, and 180 seconds after contrast material injection, respectively. MR examinations were performed using a 1.5 T MR scanner $(n=4)$ (Signa Advantage Horizon; GE Medical Systems, Milwaukee, WI, USA) with an 8-channel head coil. Pre-contrast T1-weighted spin echo images $(n=7)$ (repetition time [TR]/echo time [TE]/number of excitation [NEX], $780 \mathrm{~ms} / 8 \mathrm{~ms} / 2$ ), T2-weighted fast spin echo images $(\mathrm{n}=7)(\mathrm{TR} / \mathrm{TE} / \mathrm{NEX}, 3,600 \mathrm{~ms} / 86 \mathrm{~ms} / 1)$, with or without fat saturation, diffusion-weighted images $(n=7)$ (TR/TE/NEX, 5,200 ms $/ 70 \mathrm{~ms} / 2$, b-values $=0.800 \mathrm{~s} / \mathrm{mm}^{2}$ ), and contrast-enhanced T1-weighted images $(n=5)$, with fat saturation, were obtained from all patients after injecting 0.1 $\mathrm{mmol} / \mathrm{kg}$ gadopentetate dimeglumine. Images were obtained in at least two planes with a $5.0 \mathrm{~mm}$ slice thickness, $1.0 \mathrm{~mm}$ inter-slice gap, and $512 \times 512$ matrix.

\section{Imaging analysis}

The CT and MR images were retrospectively evaluated by two experienced radiologists who were unaware of the final diagnosis and who reached consensus. For each selected patient, the following imaging manifestations, including the location, morphology, size, number, and enhancement patterns of the lesion; change of the adjacent parotid fascia and skin; and distribution of the swollen cervical lymph nodes were further evaluated. Size was measured as the maximal diameter in the axial section and defined as pathological when it exceeded $6 \mathrm{~mm}$, based on the morphological study of normal parotid lymph nodes. The tumor location in the parotid gland was categorized into two areas: superficial lobe and deep lobe. The cervical node was defined as clinically pathological when it had a minimal axial diameter of $10 \mathrm{~mm}$ or central necrosis. The parotid parenchyma could be seen between lesion and parotid fascia. The changes in parotid fascia were compared with the contralateral side. The signal intensity of the lesion on MR images was compared with that of the masseter muscle. On post-contrast MR images, the degree of enhancement was also subjectively assessed as mild enhancement (less than or equal to that of the masseter muscle) or marked enhancement (greater than that of the masseter muscle).

\section{Pathologic analysis}

Twenty-five patients in this study were hospitalized in the two departments related to head and neck surgery, ie, Department of Oral and Maxillofacial Surgery and Department of Otorhinolaryngology. The pathologic diagnosis of parotid LNTB was established in the Department of Pathology. The surgically removed or biopsy samples were processed using 
the classical histopathological technique (formalin fixation and paraffin embedding) and then stained with H\&E.

\section{Results \\ Clinical data}

Among 25 patients (eleven males and 14 females; age range 13-76 years; mean age 39.7 years) with TB of the parotid nodes, 20 patients $(80 \%)$ presented with non-tender and slow-growing swelling in the pretragal area or below the ear for 7 days to 30 years, two patients presented with slightly tender mass, and the color and temperature of the overlying skin were red and hot in three patients (Table 1). Twenty patients $(80 \%)$ had no history of TB exposure. Only five patients $(20 \%)$ had a history of TB, including two cases of cervical tuberculous lymphadenitis, two cases of TB of the orbit, and one case of pulmonary TB (Table 1). No association was found between incidence and gender (Table 1), while younger patients (age $<50$ years) were more vulnerable to this disease (Table 1). Purified protein derivative (PPD) skin test or Mantoux test was positive in all patients who were tested $(n=9)$ (Table 1). Chest radiographs were normal in 20 patients $(80 \%)$ and five patients $(20 \%)$ showed typical

Table I Clinical data of the patients with tuberculosis of the parotid nodes

\begin{tabular}{ll}
\hline Variable & $\mathbf{n}(\mathbf{N})$ \\
\hline Age & \\
$<50$ years & $18(25)$ \\
$\geq 50$ years & $7(25)$ \\
Gender & \\
Male & $11(25)$ \\
Female & $14(25)$ \\
TB symptoms & \\
Yes & $5(25)$ \\
No & $20(25)$ \\
History of TB & \\
Yes & $5(25)$ \\
No & $20(25)$ \\
Chest X-ray & \\
$(+)$ & $5(25)$ \\
$(-)$ & $20(25)$ \\
PPD skin test & \\
$(+)$ & $9(9)$ \\
$(-)$ & $0(9)$ \\
Clinical diagnosis & \\
TB & $9(25)$ \\
Tumors & $16(25)$ \\
Imaging diagnostic accuracy (n/N) & \\
CT & $28.6 \%(6 / 2 I)$ \\
MRI & $57.1 \%(4 / 7)$ \\
\hline Abbrevions TB, thbers & \\
\hline
\end{tabular}

Abbreviations: TB, tuberculosis; PPD, purified protein derivative; $C T$, computed tomography; MRI, magnetic resonance imaging. findings of chronic TB with fibrocalcific lesions in the upper lobes (Table 1). US of the parotid area was performed in 13 patients, among whom eleven patients were considered to have neoplastic lesions and two patients swollen lymph nodes. Surgical resection of the parotid masses was performed in 22 patients $(88 \%)$, and fine needle aspiration cytology (FNAC) was performed under US guidance in three patients (12\%).

Among our cases, in only nine out of 25 cases (36\%) was there a suspicion of TB according to the clinical diagnosis (Table 1 ), and in only ten out of 25 cases (40\%) was there a suspicion of TB according to the CT and MRI. CT correctly diagnosed the nature of lesions in $28.6 \%$ of cases and MRI in $57.1 \%$ of cases. There was a trend toward slightly higher diagnostic accuracy of MRI than that of CT (Table 1), although this difference was not statistically significant. In the remaining 15 cases, the suspected diagnoses varied, including six cases of pleomorphic adenoma, five cases of Warthin's tumor, and four cases of malignant tumors.

\section{Imaging findings}

A total of 43 round, oval or irregular nodules with a maximal cross-sectional diameter of $0.6-3.2 \mathrm{~cm}$ were found in 25 patients on CT and MRI, including eleven (44\%) cases of single lesion on unilateral side, 12 (48\%) cases of multiple lesions on unilateral side, and two (8\%) cases of multiple lesions on bilateral sides. The multiple lesions accounted for $56 \%$, and unilateral lesions were arranged linearly up and down the parotid glands (Figure 1A, B). Among the 43 lesions, 38 (88.4\%) lesions involved the superficial lobe and five (11.6\%) lesions involved the deep lobe. There were four types of changes in the parotid fascia: local thickening $(40 \%, \mathrm{n}=10)$; local rupture with thickened adjacent skin $(28 \%, n=7)$; focal bulge $(20 \%$, $\mathrm{n}=5)$; and no changes $(12 \%, \mathrm{n}=3)$ (Figure $1 \mathrm{C}, \mathrm{D})$. Cervical lymphadenopathy was seen in 14 out of 25 patients $(56 \%)$ on CT and MRI, while six patients (24\%) had involvement of bilateral lymph nodes. Among the 14 patients, the most commonly affected areas of lymph nodes were level IIb $(\mathrm{n}=11,78.6 \%)$, level IIa $(\mathrm{n}=9,64.3 \%)$, and level III $(\mathrm{n}=7$, $50 \%$ ) (Figure 2A). The distribution of the cervical lymphadenopathy was shown in Table 2 .

The lesions were enhanced after injection of the contrast medium in four patterns on CT: homogeneous enhancement $(\mathrm{n}=13,37.1 \%)$ (Figure 2A), thick-walled ring enhancement $(\mathrm{n}=5,14.2 \%)$ (Figure 2B), garland-like enhancement $(\mathrm{n}=4$, $11.4 \%$ ) (Figure 2C), and irregular enhancement ( $n=13,37.1 \%)$ (Figure 2D). One of the lesions contained central calcification. A total of 14 lesions were found in seven patients on MR 

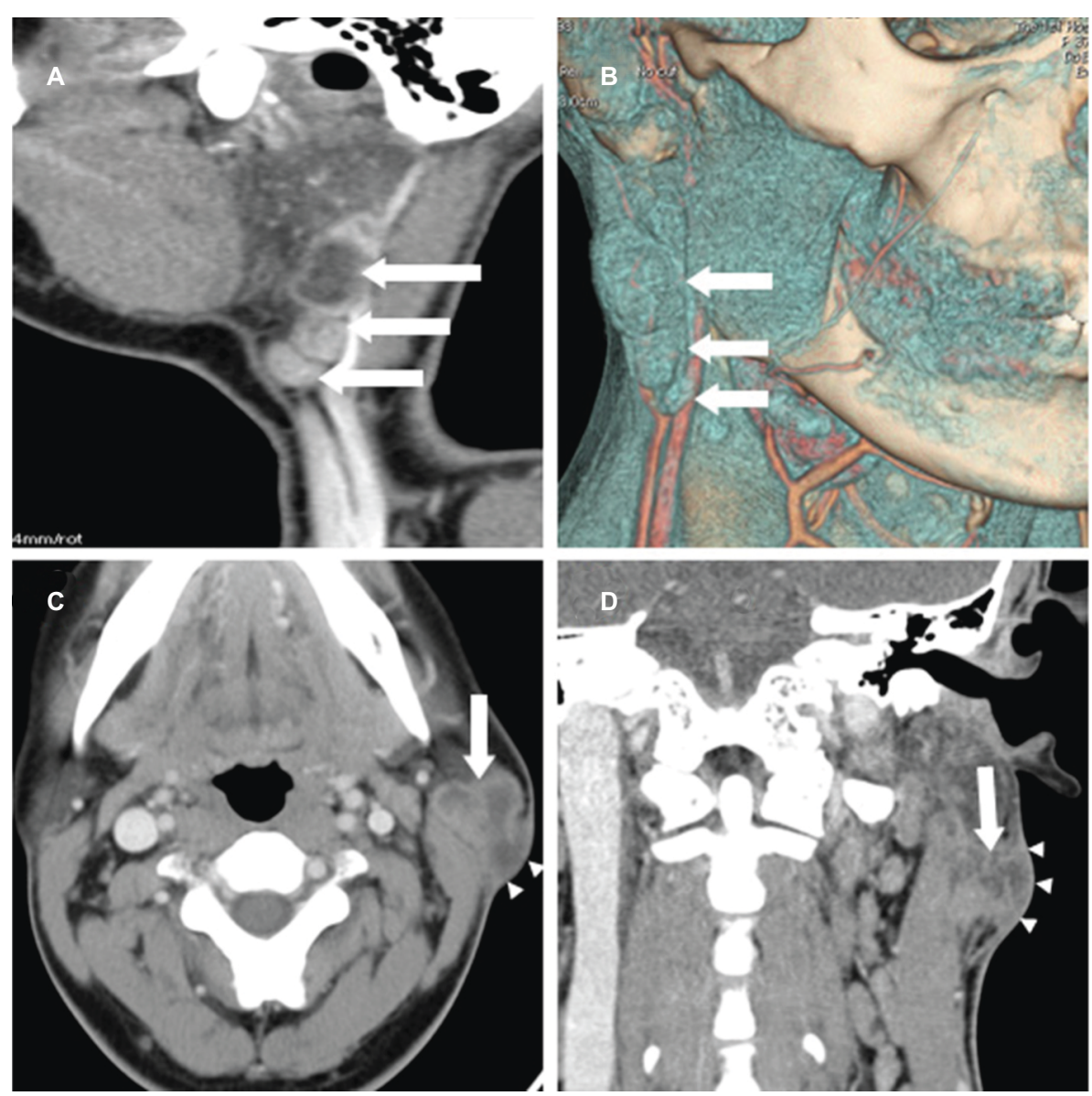

Figure I The multiple tuberculous lesions of right parotid nodes are linearly arranged on sagittal CT image.

Notes: Tuberculosis of the left parotid lymph node in a 28-year-old female. (A) Volume rendering image (white arrow); (B) local rupture of parotid fascia and thickened adjacent skin (white arrow). Axial and coronal images (C, D) show an irregular cyst-like enhanced mass (white arrow), located in the superficial lobe and close to the surface of the sternocleidomastoid which involves parotid fascia; the neighboring skin is thickened and adhered (C, D) (white arrowhead).

Abbreviation: CT, computed tomography.

images (Table 3). All of the lesions mostly showed homogeneous isointense signal intensity on T1-weighted image (T1WI) (n=7) (Figures 3A). In contrast, the lesions showed five cases for hyperintense, one case for slightly hyperintense, and one case for isointense signal intensity on T2-weighted image (T2WI) with or without fat saturation $(\mathrm{n}=7)$ (Figure 3B). Post-contrast MR images demonstrated areas of homogeneous and obvious enhancement or heterogeneous enhancement $(\mathrm{n}=5)$ (Figure 3C, D). Surrounding parotid parenchyma edema was displayed clearly in five patients and enhanced obviously (Figure 3C, D). Thickened adjacent skin was found in four cases, including sinus tract between the lesion and skin in one case. The ipsilateral parotid fascia changes could be seen distinctly on coronal T1WI and T2WI without fat saturation compared with the contralateral fascia (Figure 4A, B). The multiple lesions were linearly arranged, which could be shown clearly on coronal and sagittal scanning, as well as the cervical lymphadenopathy (Figure 4C, D). Most of the lesions showed homogeneous isointense signal on T1WI and hyperintense signal on T2WI with fat saturation (Figure 5A, B). However, markedly hyperintense signal intensity was shown on diffusion-weighted imaging (DWI) $(n=6)$ (Figure 5C) and low signal intensity on corresponding apparent diffusion coefficient (ADC) map (Figure 5D).

\section{Pathologic findings}

Among all patients with TB of the parotid nodes, specimens of 25 patients were obtained surgically or by biopsy. The histology showed granulomatous inflammation: tuberculous nodules (Figure 6A) with Langhans giant cells (Figure 6B, C), epithelioid macrophages (Figure 6C), and caseous necrosis (Figure 6D).

\section{Discussion}

TB remains one of the most common infectious diseases and a global health problem. ${ }^{15}$ At the same time, TB diagnosis is 

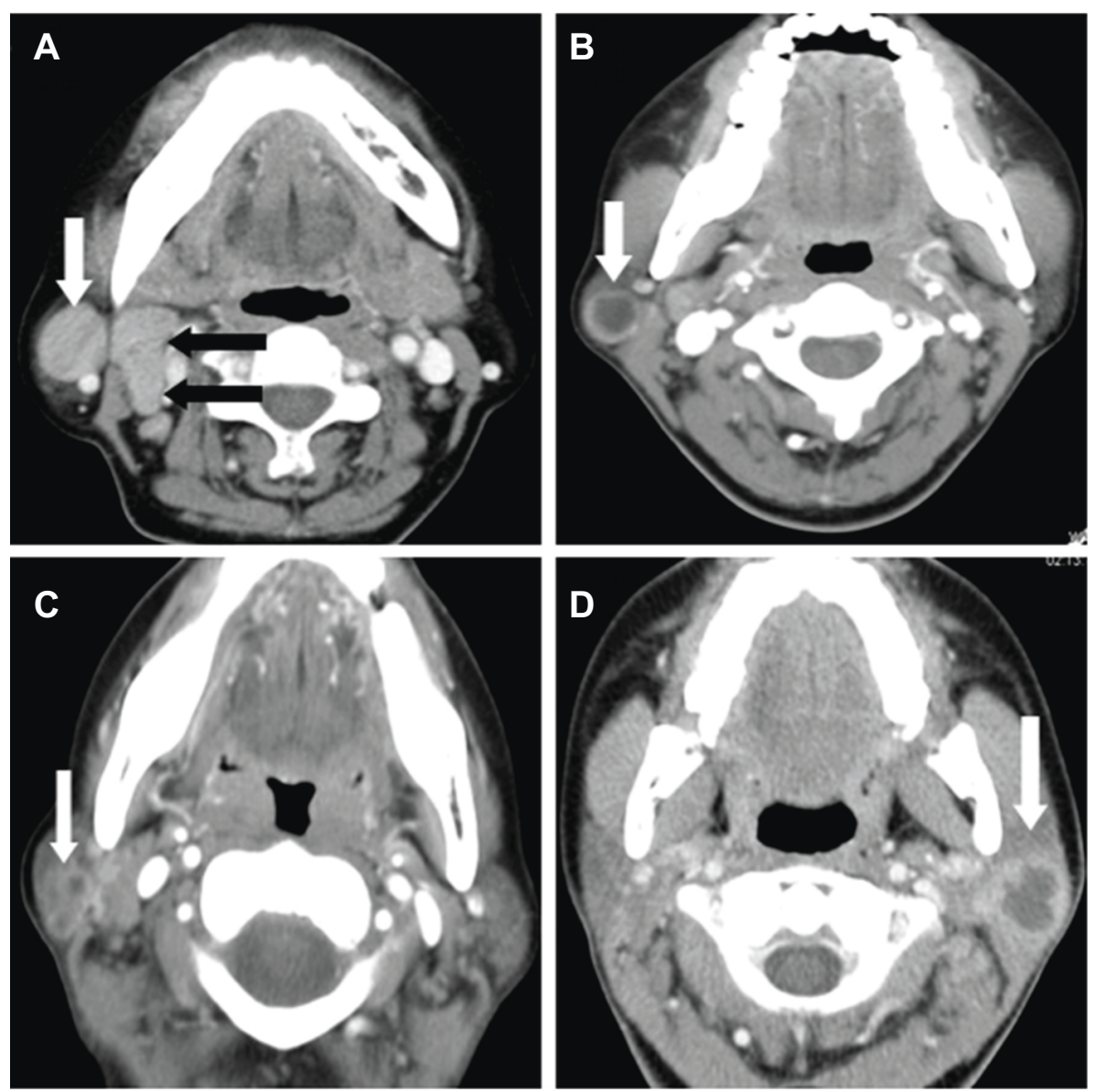

Figure 2 Homogenous enhancement.

Notes: Tuberculosis of the right parotid node in a 30-year-old female. Axial CT image (A) shows a regular mass (white arrow) with homogeneous enhancement, which is located in the superficial lobe, accompanied by cervical lymphadenopathy level lla and level llb (black arrow). Thick-walled circular enhancement. Tuberculosis of the right parotid nodes in a 37-year-old female. Axial CT image (B) shows a smooth rounded nodule (white arrow) with thick-walled circular enhancement and well-defined border, which is located in the superficial lobe. Garland-like enhancement. Tuberculosis of the right parotid lymph nodes in a 37-year-old female. Axial CT image (C) shows a lobulated nodule (white arrow) with garland-like enhancement, which is located in the superficial lobe. Irregular cyst-like enhancement. Tuberculosis of the left parotid lymph nodes in a 16-year-old female. Axial CT image (D) shows a mass (white arrow) with irregular cyst-like enhancement, which is located in the superficial lobe.

Abbreviation: $\mathrm{CT}$, computed tomography.

Table 2 Distribution of cervical lymphadenopathy $(n=25)$

\begin{tabular}{lll}
\hline Lymph node & Ipsilateral & Contralateral \\
\hline la & I (4\%) & $2(8 \%)$ \\
Ib & I (4\%) & $4(16 \%)$ \\
Ila & $9(36 \%)$ & $5(20 \%)$ \\
IIb & II (44\%) & $4(16 \%)$ \\
III & $7(28 \%)$ & $4(16 \%)$ \\
IV & I (4\%) & $2(8 \%)$ \\
Va & $3(I 2 \%)$ & I (4\%) \\
Vb & $2(8 \%)$ & 0 \\
VI & 0 & 0 \\
VII & 0 & 0 \\
\hline
\end{tabular}

not always easy because of variable and nonspecific presentations. TB of the salivary gland is rare even in countries where TB is widespread and tuberculous involvement of the parotid lymph node is extremely rare. ${ }^{6-8}$ Diagnosis is usually made only after surgery or biopsy. The infection often presents clinically as a unilateral slow-growing, painless or slightly painful swelling. ${ }^{13,16}$ The color and temperature of the overlying skin are normal. These characteristics are quite different from the infection caused by common bacteria which mainly give rise to redness, swelling, and hot pain. When the capsules of the involved lymph nodes are broken, preauricular fistulas may be present. Among all patients with TB of the parotid node in our study, most presented with non-tender, slow-growing lesions in the pretragal area or under the ear, and had no systemic symptoms or history of TB exposure, which is similar to parotid tumors. PPD skin test was positive in all our patients who were tested. A positive PPD skin test may suggest a TB infection as clinical identification but cannot be relied upon. ${ }^{17,18}$

Parotid TB mainly involves the parotid lymph nodes, while the parenchyma is rarely involved..$^{19,20}$ In our study, 


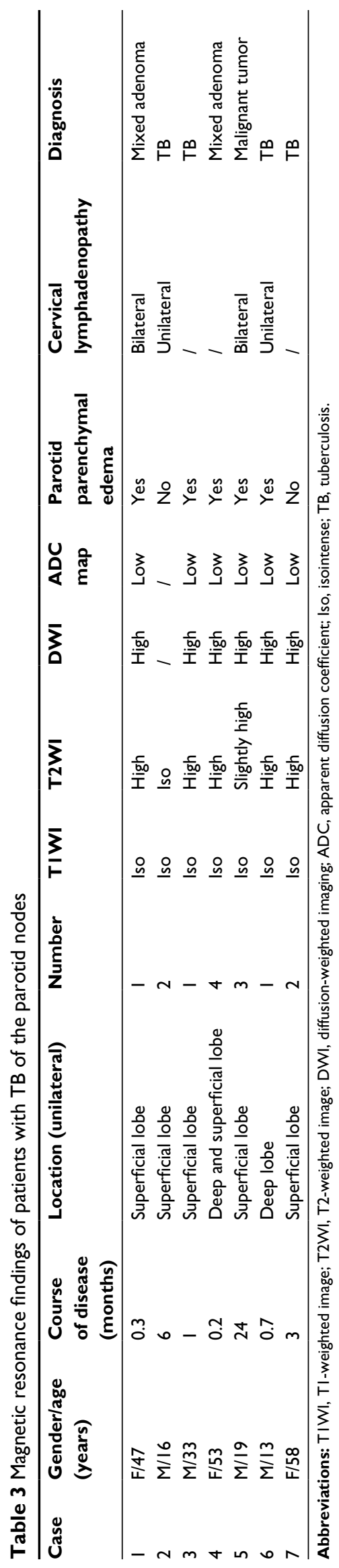

TB of the parotid nodes were most frequently noted in the superficial lobe, which corresponds well to the distribution of the parotid lymph nodes. Most nodes exist along the course of the retromandibular vein and are predominantly located in the parotid superficial lobe. ${ }^{21,22}$ However, the source of infection, mode of acquisition, and route of spread remain uncertain. ${ }^{9}$ The infection may initially involve the tonsils or gingival sulcus, spread to the parotid glands via their ducts, and then extend to the cervical nodes through the lymphatic vessels. ${ }^{13}$ Hematogenous or lymphatic spread from the lungs is another possible route. ${ }^{23}$

The characteristic histopathological feature of TB is tubercle accompanied by different degrees of caseous necrosis. The tubercle is formed by epithelioid macrophages, Langhans giant cells, lymphocytes as well as a small amount of reactive hyperplasia of fibroblasts surrounding the caseous centers. ${ }^{24}$ This was in accord with our study.

Surgical resection of the parotid lesions was performed in 22 patients in our study. And, 13 patients (most of them less than 50 years old) underwent superficial parotidectomy, which would cause physical and mental injury to young patients. FNAC was performed in three patients who avoided the surgery, and the lesions gradually disappeared due to a standard antitubercular regimen. FNAC is recommended as a useful and reliable technique for the diagnosis of parotid TB. ${ }^{6,9}$ It has a high sensitivity and specificity in the diagnosis of TB. Handa et al reported five cases of parotid TB documented by FNAC and medically treated. ${ }^{13}$ When the diagnosis is obtained by minimally invasive methods, surgery becomes unnecessary and antituberculous chemotherapy is sufficient to obtain resolution.

Diagnosing parotid TB remains challenging, as clinical findings are useful but still have limitations in differentiating TB of parotid nodes from tumors. Therefore, imaging has assumed an important role in planning a cure for assessing the location and features of parotid TB. US as a first line of investigation is particularly advantageous for assessing the parotid gland, due to this organ being superficial. However, US is operator-dependent, and assessment of deep structures, such as the deep lobe of the parotid gland and the relationship with the facial nerve, is suboptimal. ${ }^{25,26} \mathrm{And}$, none of our cases were diagnosed correctly by US. Recent advancements in CT imaging, such as isotropic imaging and multiplanar reconstructions, have improved the utility of CT in the assessment of parotid lesions. MRI had been considered as the technique of choice for parotid gland study, on account of its accuracy in soft tissue evaluation and multi-planarity. However, only ten out of 25 cases were 

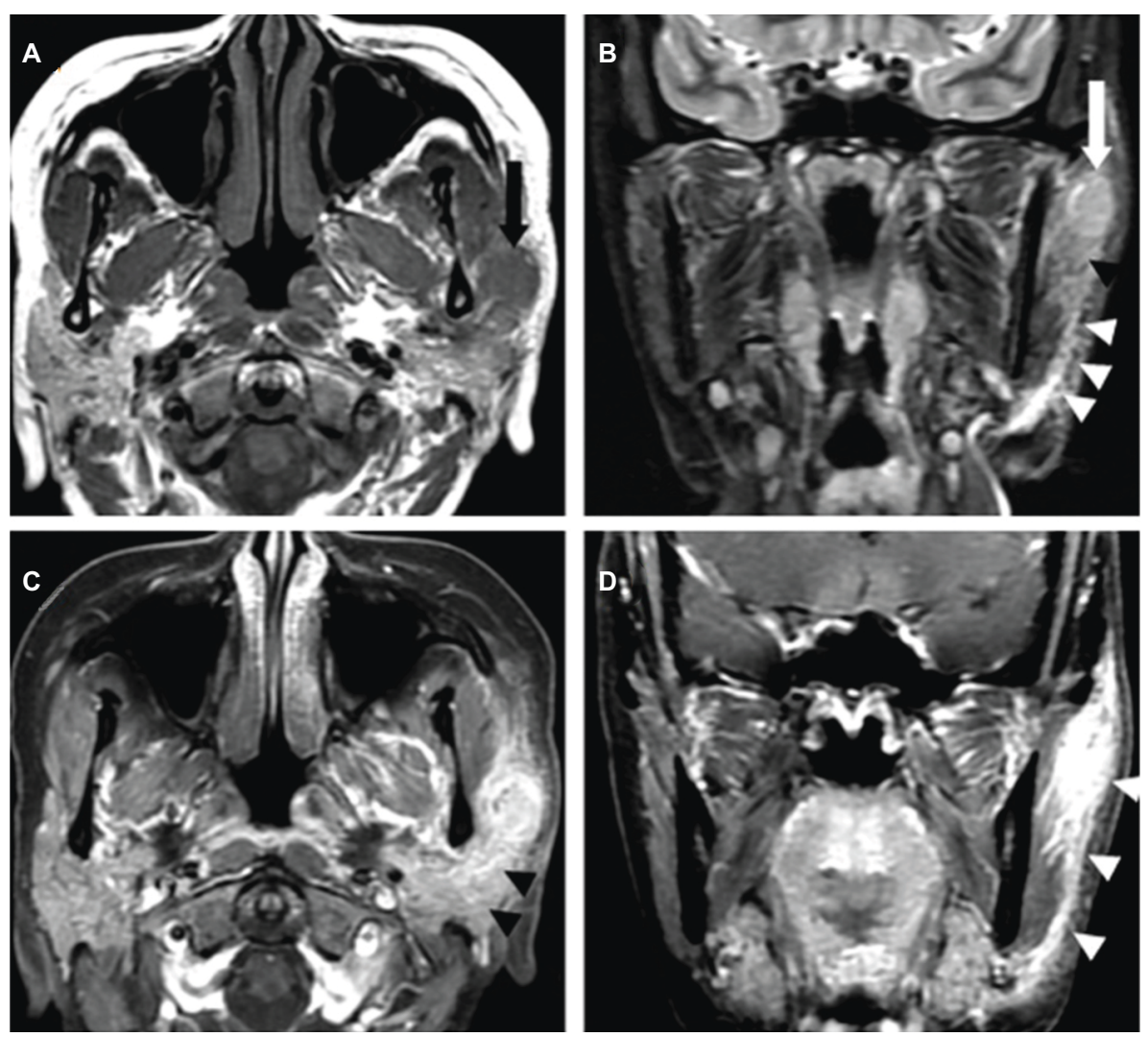

Figure 3 Tuberculosis of the left parotid lymph node in a 19-year-old male.

Notes: The lesion is located in the superficial lobe, with isointensity on axial TIWI (A) (black arrow), slight hyperintensity on coronal T2WI-FS (B) (white arrow), and obvious enhancement (C, D). The surrounding parotid parenchymal edema (black arrowhead) and thickened adjacent fascia (white arrowhead) are shown clearly on coronal T2WI-FS (B) and obvious enhancement is shown on post-contrast images (C, D).

Abbreviations: TIWI, TI-weighted image; T2WI-FS, T2-weighted image with fat saturation.

correctly diagnosed according to CT and MRI in our study. TB of the parotid nodes always mimic parotid tumor, so it is usually misdiagnosed as a tumor clinically and radiologically before surgery. Previous literature has described tuberculous lymphadenitis typically manifested as multi-loculated nodal mass with thick smooth-walled enhancing rims and central lucency or low-density central mass with thick corrugated enhancing rim. ${ }^{11,12}$ In our study, we found four patterns of enhancement on CT, while homogeneous enhancement and irregular enhancement were the most common patterns revealed in 26 lesions. Furthermore, 52\% of patients $(n=13)$ had multiple lesions. Unilateral multiple lesions were arranged linearly up and down the parotid gland on coronal or sagittal images, which may be due to the infection spreading along the lymphatic drainage within the parotid gland. We also found that the ipsilateral parotid fascia of most patients had changed. It may be related to the inflammatory reaction depending on the functional state of the patient's immune system. Cervical lymphadenopathy was seen in 14 out of 25 patients. Level IIb, level IIa, and level III were the most commonly affected areas. The cervical lymphadenopathy probably proved the hypothesis that the infection spreads along regional lymphatic drainage from an insidious source in the vicinity and appeared to be consistent with previously reported lymphatic drainage patterns of the head and neck. ${ }^{27}$ However, primary parotid malignancy is seldom accompanied by cervical lymph node metastasis. ${ }^{28} \mathrm{~A}$ clinical case report has described the signal intensity of parotid TB on MRI as hypointense on T1WI and hyperintense on T2WI with homogeneous enhancement. ${ }^{9}$ In our study, the signal intensity of lesions was isointense on T1WI, which was manifested clearly compared with the surrounding hyperintensity of the parotid parenchyma. On T2WI, five cases showed hyperintensity, one case slight hyperintensity, and one case isointensity. Another interesting finding was that two patients with lower signal intensity on T2WI had a longer course of disease than the other five patients. Thus, further studies are needed to confirm the relation between the course of disease and T2 signal intensity. High signal intensity was shown on DWI and low signal intensity on ADC map. These findings probably reflect inflammatory cell infiltration in these lesions; however, this 

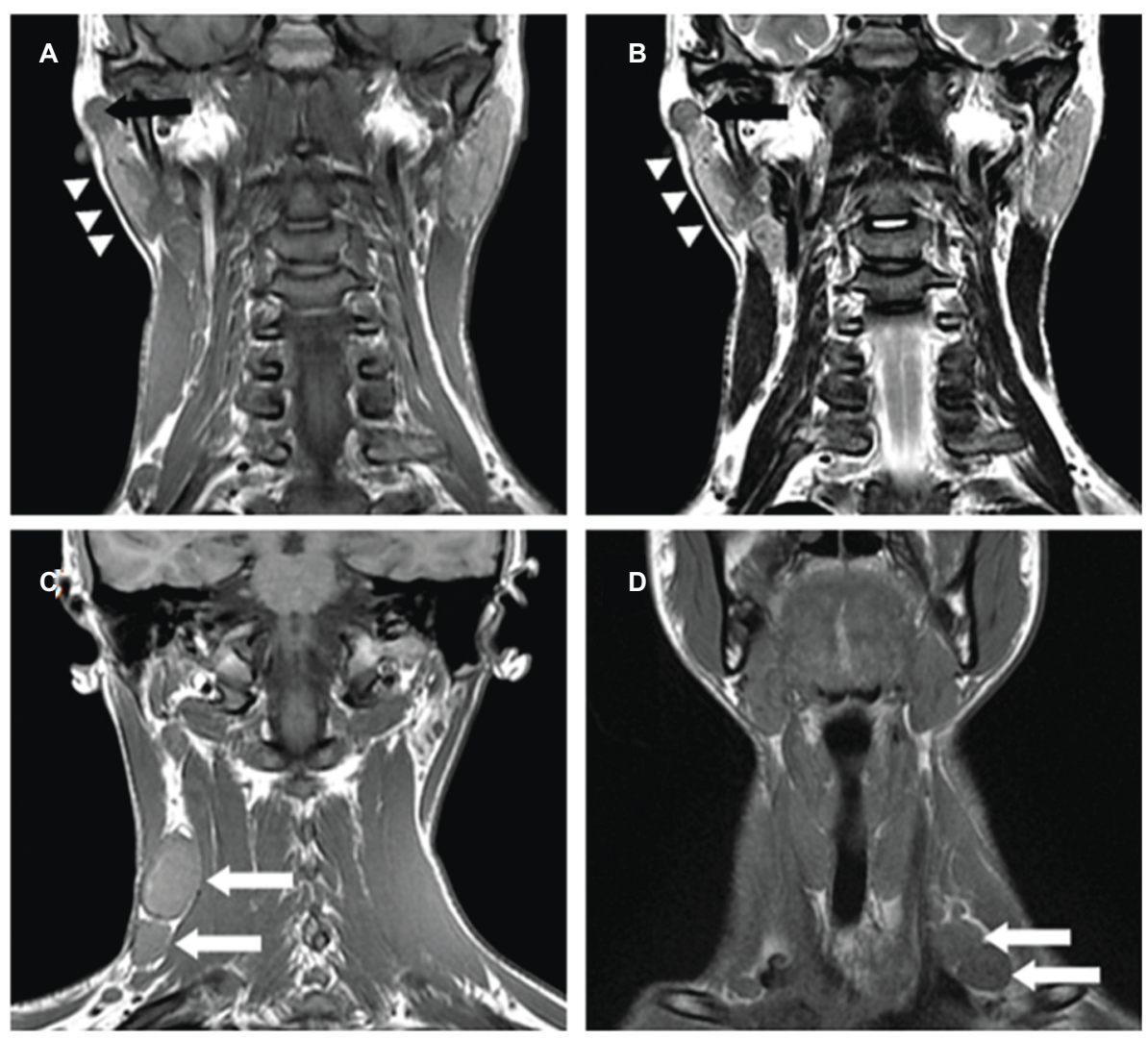

Figure 4 Tuberculosis of the right parotid lymph node in a 16-year-old male.

Notes: Coronal TIWI (A) shows a nodule with isointense signal intensity (black arrow), which is located in the superficial lobe. The lesion also shows isointense signal intensity (black arrow) on coronal T2WI (B). Bilateral cervical lymphadenopathy is shown on coronal TIWI (C, D) (white arrow). The thickened parotid fascia is shown clearly on coronal TIWI and T2WI (A, B) (white arrowhead).

Abbreviations: TIWI, TI-weighted image; T2WI, T2-weighted image.

result deserves a deeper analysis in a larger population. These findings mimic those found in patients with parotid malignancy. Previous radiological literature has suggested ADC value and magnetic resonance spectrum (MRS) may be helpful in differentiation of chronic infection from malignancy. ${ }^{29}$ It is a pity we did not survey ADC value and apply MRS in this study, so more studies are required on daily clinical application. We also found surrounding parotid parenchymal edema in five out of seven patients on MRI due to its accuracy in soft tissue evaluation and multiple parameters, which is the typical and common manifestation of the inflammatory infiltration. However, the edema was less likely to be shown on CT scan. Likewise, the changes in the parotid fascia were manifested clearly on MRI, especially on coronal T2WI and T1WI without fat saturation in our study.

Our present study provided only some CT and MR findings for TB of the parotid nodes, and some findings overlap with the findings for a number of primary benign and malignant tumors. The most commonly misdiagnosed diseases in our study were pleomorphic adenoma, Warthin tumor, and malignant tumors such as metastasis and mucoepidermoid carcinoma. Pleomorphic adenoma is the most common parotid tumor. It typically appears as a smoothly marginated or lobulated homogeneous mass, shows prominent enhancement, and demonstrates delayed enhancement. ${ }^{30,31}$ The high signal intensity on T2WI and strong enhancement after contrast administration are wellknown specific MRI findings of it. ${ }^{32}$ Warthin tumor is the second most common benign parotid tumor and the typical location is the parotid tail..$^{33}$ Older age, bilaterality, smoking, and male sex have shown a significant association with Warthin tumor. ${ }^{34}$ After using the contrast agent, Warthin tumors show significant enhancement in arterial phase and recede sharply in delayed phase. The characteristics of metastases to the parotid nodes have been reported as a solitary mass located in the parotid tail or superficial lobe, and cervical nodes metastasis. ${ }^{35}$ So, it is difficult to distinguish metastases to the parotid nodes through routine contrast $\mathrm{CT}$ and MRI because the imaging features may mimic TB. However, some specific characteristic clinical 

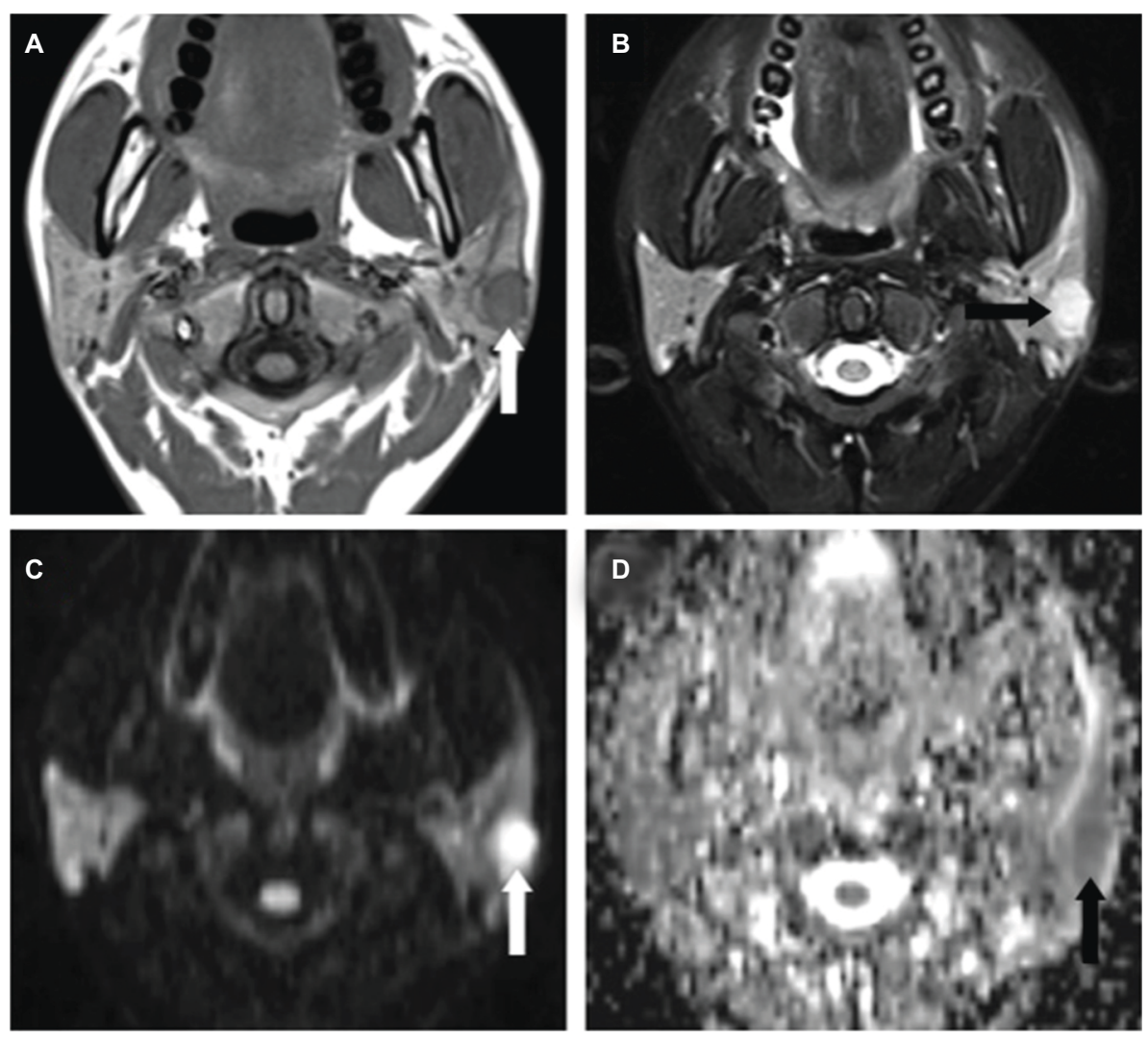

Figure 5 Tuberculosis of the right parotid lymph node in a 58 -year-old female

Notes: The lesion is located in the superficial lobe with isointense signal intensity on axial TIWI (A) (white arrow) and heperintense signal intensity on axial T2WI-FS images (B) (black arrow). Markedly hyperintense signal intensity is shown on DWI (C) (white arrow) and hypointense signal intensity on ADC map (D) (black arrow).

Abbreviations: TIWI, TI-weighted image; T2WI-FS, T2-weighted image with fat saturation; DWI, diffusion-weighted imaging; ADC, apparent diffusion coefficient.
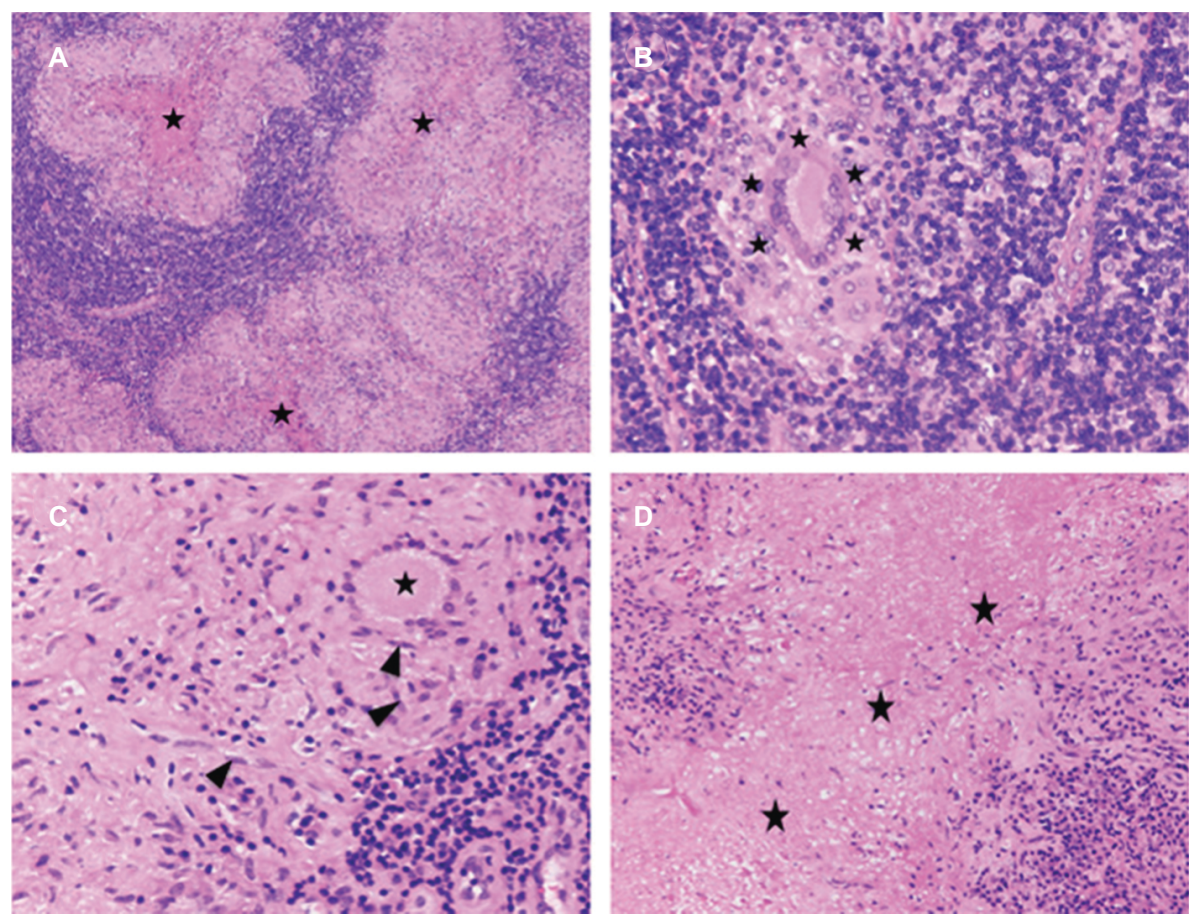

Figure 6 Histopathology of parotid nodes.

Notes: Tuberculous nodules (A) (black star) with typical Langhans giant cell (B, C) (black star), epithelioid macrophages (C) (black arrowhead), and caseous necrosis (D) (black star). (A: H\&E stain, magnification $\times 100$; B, C: H\&E stain, magnification $\times 400$; D: H\&E stain, magnification $\times 200$ ). 
and imaging features such as younger age, multiple lesions, and calcification may be helpful in determining differential diagnosis. Advanced MR imaging such as DWI, MRS, and dynamic contrast-enhanced MRI (DCE-MRI) may aid in this differentiation..$^{30,36-38}$ Our data may, thus, suggest that further investigations are warranted.

MRI is the first-line study for the evaluation of parotid lesions, especially when there is a strong suspicion that the lesion is neoplastic. It was shown to be more effective than $\mathrm{CT}$ in the manifestation of the surrounding tissue edema. Moreover, as it is radiation-free, MRI is more suitable for re-examination after chemotherapy to establish curative effect including size and signal change. However, the advantages of CT include the ability to show calcification, the lower cost, and shorter examination time. It is the method of choice in patients with contraindications for MR imaging.

Our study had several limitations. First, our study was performed retrospectively. Second, we analyzed a relatively small dataset, thus limiting the generalizability of our results. Third, our standard protocol did not include ADC value, DCE-MRI, and MRS sequences, which could add additional information in order to differentiate TB from tumors of the parotid gland. Due to these considerations, further validation studies in a larger population, preferably in a prospective study, can improve the reproducibility and robustness of our findings.

In summary the present work suggested that $\mathrm{TB}$ of the parotid nodes is an uncommon entity which always mimics tumor clinically and radiologically. Its diagnosis should be considered when slow-growing parotid swellings are multiple, located in the superficial lobe, and accompanied by surrounding tissue edema, parotid fascia changes, and cervical lymphadenopathy, especially in younger (age <50 years) patients with positive PPD skin test. Correct radiological interpretation can provide clues toward tubercular pathology. It can be easily diagnosed by FNAC and treated by a standard antitubercular therapy regimen. At last, surgical explorations will be avoided.

\section{Acknowledgments}

This study was supported by the Medical Research Key Program of the National Health and Family Planning Commission of Chongqing, China (no 20141016 and no 2016ZDXM026) and the Basic and Frontier Research Project of Chongqing, China (no cstc2016jcyjA0294). The authors thank EzPubedit for linguistic assistance during the preparation of this manuscript.

\section{Disclosure}

The authors report no conflicts of interest in this work.

\section{References}

1. Karim MM, Chowdhury SA, Hussain MM, Faiz M. A clinical study on extrapulmonary tuberculosis. J Bangladesh Coll Phys Surg. 2006;24:19-28.

2. Gopal R, Padmavathy BK, Vasanthi S, Jayashree K. Extrapulmonary tuberculosis: a retrospective study. Indian JTuberc. 2001;48(4):225-226.

3. Dandapat MC, Mishra BM, Dash SP, Kar PK. Peripheral lymph node tuberculosis: a review of 80 cases. Br J Surg. 1990;77(8):911-912.

4. Subrahmanyam M. Role of surgery and chemotherapy for peripheral lymph node tuberculosis. Br J Surg. 1993;80(12):1547-1548.

5. Kato M, Kinoshita T. Tuberculous lymphadenitis. Nihon Rinsho. 1998;56(12):3122-3125.

6. Lee IK, Liu JW. Tuberculous parotitis: case report and literature review. Ann Otol Rhinol Laryngol. 2005;114(7):547-551.

7. Hamdan AL, Hadi U, Shabb N. Tuberculous parotitis: a forgotten entity. Otolaryngol Head Neck Surg. 2002;126(5):581-582.

8. Vyas S, Kaur N, Yadav TD, Gupta N, Khandelwal N. Tuberculosis of parotid gland masquerading parotid neoplasm. Natl J Maxillofac Surg. 2012;3(2):199-201.

9. Birkent H, Karahatay S, Akcam T, Durmaz A, Ongoru O. Primary parotid tuberculosis mimicking parotid neoplasm: a case report. J Med Case Rep. 2008;2:62.

10. Chou YH, Tiu CM, Liu CY, et al. Tuberculosis of the parotid gland: sonographic manifestations and sonographically guided aspiration. J Ultrasound Med. 2004;23(10):1275-1281.

11. Wei Y, Xiao J, Pui MH, Gong Q. Tuberculosis of the parotid gland: computed tomographic findings. Acta Radiol. 2008;49(4):458-461.

12. Silvers AR, Som PM. Salivary glands. Radiol Clin North Am. 1998; 36(5):941-966.

13. Handa U, Kumar S, Punia RS, Mohan H, Abrol R, Saini V. Tuberculous parotitis: a series of five cases diagnosed on fine needle aspiration cytology. J Laryngol Otol. 2001;115(3):235-237.

14. Erdoğan B, Uzaslan E, Demirdöğen E, Balaban Adim S, Salan A, Cakir U. An unusual reason of parotid gland enlargement; parotid gland tuberculosis. Tuberk Toraks. 2006;54(2):182-184.

15. World Health Organization. Global Tuberculosis Report 2018. Geneva: World Health Organization; 2018. Available from: http://www.who.int/ tb/publications/global_report/en/. Accessed September 26, 2018.

16. Iserì $\mathrm{M}$, Aydìner $\mathrm{O}$, Celik L, Peker O. Tuberculosis of the parotid gland. J Laryngol Otol. 2005;119(4):311-313.

17. Robson CD. Imaging of granulomatous lesions of the neck in children. Radiol Clin North Am. 2000;38(5):969-977.

18. Robson CD, Hazra R, Barnes PD, Robertson RL, Jones D, Husson RN. Nontuberculous mycobacterial infection of the head and neck in immunocompetent children: CT and MR findings. AJNR Am J Neuroradiol. 1999;20(10):1829-1835.

19. Ozcan C, Apa DD, Aslan G, Gülhan S, Görür K. Mycobacterium tuberculosis infection within parotid gland Warthin tumor. J Craniofac Surg. 2008;19(6):1561-1565.

20. Dixit R, Shah V, Dixit K, Solanki RN, Kotadia J. Tuberculous abscess of parotid gland. J Ind Acad Clin Med. 2005;6:161-163.

21. Thom JJ, Moore EJ, Price DL, Kasperbauer JL, Starkman SJ, Olsen KD. The Role of Total Parotidectomy for Metastatic Cutaneous Squamous Cell Carcinoma and Malignant Melanoma. JAMA Otolaryngol Head Neck Surg. 2014;140(6):548-554.

22. Harada H, Omura K. Metastasis of oral cancer to the parotid node. Eur J Surg Oncol. 2009;35(8):890-894.

23. Kundu S, Das S, Dey A, Sengupta A. Tuberculosis of parotid gland - a rare clinical entity. Indian J Otolaryngol Head Neck Surg. 2004;56(1):57-58.

24. Kim YH, Jeong WJ, Jung KY, Sung MW, Kim KH, Kim CS. Diagnosis of major salivary gland tuberculosis: experience of eight cases and review of the literature. Acta Otolaryngol. 2005;125(12):1318-1322. 
25. Lee YY, Wong KT, King AD, Ahuja AT. Imaging of salivary gland tumours. Eur J Radiol. 2008;66(3):419-436.

26. Kei PL, Tan TY. CT "invisible" lesion of the major salivary glands a diagnostic pitfall of contrast-enhanced CT. Clin Radiol. 2009;64(7):744-746.

27. Suton P, Lukšić I, Müller D, Virag M. Lymphatic drainage patterns of head and neck cutaneous melanoma: does primary melanoma site correlate with anatomic distribution of pathologically involved lymph nodes? Int J Oral Maxillofac Surg. 2012;41(4):413-420.

28. Christe A, Waldherr C, Hallett R, Zbaeren P, Thoeny H. MR imaging of parotid tumors: typical lesion characteristics in MR imaging improve discrimination between benign and malignant disease. AJNR Am J Neuroradiol. 2011;32(7):1202-1207.

29. Yu Q, Yang J, Wang P. Malignant tumors and chronic infections in the masticator space: preliminary assessment with in vivo singlevoxel 1H-MR spectroscopy. AJNR Am J Neuroradiol. 2008;29(4): 716-719.

30. Thoeny HC. Imaging of salivary gland tumours. Cancer Imaging. 2007;7:52-62.

31. Lev MH, Khanduja K, Morris PP, Curtin HD. Parotid pleomorphic adenomas: delayed CT enhancement. AJNR Am J Neuroradiol. 1999;19(10):1835-1839.
32. Mortelé B, Lemmerling M, Seynaeve P, Clarysse P, Quintens F, Kunnen M. Hemangiopericytoma of the parotid gland: CT and MR features. Eur Radiol. 2001;11(6):1073-1075.

33. Yousem DM, Kraut MA, Chalian AA. Major salivary gland imaging. Radiology. 2000;216(1):19-29.

34. Sadetzki S, Oberman B, Mandelzweig L, et al. Smoking and risk of parotid gland tumors: a nationwide case-control study. Cancer. 2008;112(9):1974-1982.

35. Kashiwagi N, Murakami T, Toguchi M, et al. Metastases to the parotid nodes: CT and MR imaging findings. Dentomaxillofac Radiol. 2016;45(8):20160201.

36. Wang P, Yang J, Yu Q, Ai S, Zhu W. Evaluation of solid lesions affecting masticator space with diffusion-weighted MR imaging. Oral Surg Oral Med Oral Pathol Oral Radiol Endod. 2010;109(6):900-907.

37. Razek AA, Elsorogy LG, Soliman NY, Nada N. Dynamic susceptibility contrast perfusion MR imaging in distinguishing malignant from benign head and neck tumors: a pilot study. Eur J Radiol. 2011;77(1): 73-79.

38. Liu S, Zhen F, Sun N, et al. Apparent diffusion coefficient values detected by diffusion-weighted imaging in the prognosis of patients with locally advanced esophageal squamous cell carcinoma receiving chemoradiation. Onco Targets Ther. 2016;9:5791-5796.
Infection and Drug Resistance

\section{Publish your work in this journal}

Infection and Drug Resistance is an international, peer-reviewed openaccess journal that focuses on the optimal treatment of infection (bacterial, fungal and viral) and the development and institution of preventive strategies to minimize the development and spread of resistance. The journal is specifically concerned with the epidemiology of antibiotic

\section{Dovepress}

resistance and the mechanisms of resistance development and diffusion in both hospitals and the community. The manuscript management system is completely online and includes a very quick and fair peerreview system, which is all easy to use. Visit http://www.dovepress.com/ testimonials.php to read real quotes from published authors. 